\title{
Preface to the QICS special issue
}

\author{
Jerry Blackford, Toru Sato, Jun Kita, Henrik Stahl
}

We are delighted to bring together a suite of papers arising from the "QICS" (Quantifying and monitoring potential ecosystem impacts of geological carbon storage) project. Carbon Capture and Storage (CCS) is poised to play a major role in reducing harmful greenhouse gas emissions as the world transforms to a low carbon economy. In addition to the challenge to develop economically viable CCS is the regulatory requirement for monitoring to detect leakage (or demonstration of reservoir integrity) and an assessment of environmental impact, should leakage occur. The QICS project addressed offshore geological storage and asked two main questions: 1) if leakage occurred would the environmental impact to the marine ecosystem be significant? and 2) what are the best methods for detection and impact monitoring in the marine environment? To address these questions the project undertook a novel injection of $\mathrm{CO}_{2}$ into marine sediments in order to mimic, as realistically as possible, leakage at the sea floor.

This real world manipulation pioneered by QICS has proved to be a highly effective research mechanism. Our key questions require a consideration of the full complexity of the natural system, which cannot be replicated in laboratory situations. Ecosystem impact can only be assessed within the context of natural spatial and temporal dynamics of communities including species interactions and behaviour. Monitoring techniques can only be adequately tested within the context of heterogeneous sediments and tidally driven hydrodynamics which fundamentally affect the relationship between the amount of $\mathrm{CO}_{2}$ entering the system and the observable chemical or acoustic perturbations. Such experiments, although complex, are recommended for future studies assessing a range of marine impact questions.

The papers collected here describe the outcomes of this very multidisciplinary research, as well as describing the complex methodology (Taylor, Stahl et al.) and the social perception issues (Mabon et al.) relating to the experiment. The first set of papers address the dynamics and detectability of gas in the sediments (Cevatoglu et al.) and water column (Sellami et al.; Dewar et al.; Berges et al.) using a variety of acoustic, observational and modelling approaches. A number of papers describe the biochemical perturbation observed in sediments and water column (Lichtschlag et al.; Taylor, Lichtschlag et al.; Tsukasaki et al.) and discuss chemical detection methodologies (Queiros et al.; Atamanchuk et al.; Shitashima et al.; Maeda et al.) using a range of probes, sensors and analytical techniques. Hydrodynamic models have also been developed to track and quantify $\mathrm{CO}_{2}$ in the water column (Mori et al.). A group of papers discuss the biological impact (Watanabe et al.; Tait et al.; Widdicombe et al.; Pratt et al.; Kita et al.) assessing both microbes, macrofauna and megafauna using traditional, genomic and video techniques. Finally papers extrapolate the findings to an assessment of the impact potential of larger scale leakage (Phelps et al.) and to the design of baseline and monitoring programmes (Blackford et al.). In addition to these scientific papers, summaries of the projects findings aimed at a general audience can be found on the project website: www.qics.co.uk.

The project reached two broad conclusions. Firstly, environmental impacts from small scale leakage will be minimal and not ecologically significant, although in the unlikely event of larger leaks, impact could be locally more significant. Secondly, detection of small scale leakage and monitoring of impact will be challenging due to the complexity of $\mathrm{CO}_{2}$ flows and ecosystem heterogeneity but is tractable given development of existing tools, monitoring strategies and a comprehensive understanding of natural variability. 